\title{
Functions of essential nutrition for high quality spermatogenesis
}

\author{
Yunsang Cheah, Wanxi Yang* \\ The Sperm Laboratory, College of Life Sciences, Zhejiang University, Hangzhou, China. \\ E-mail: *wxyang@spermlab.org
}

Received 31 May 2011; revised 20 June 2011; accepted 5 July 2011.

\begin{abstract}
The process of sperm production is well understood, but the studies of essential nutritional elements which are necessary for successful spermatogenesis are not deeply studied as yet. Our review focuses on integrating available information of various nutritional elements involved in spermatogenesis, sperm maturation and male reproductive system development, such as Zinc, Selenium, Folate, Vitamins and others. Antioxidants protect sperm from further oxidative damage during the entire sperm production. Other nutrients assist to improve sperm quality through different ways. The important roles of macronutrients like lipids, amino acids and proteins are emphasized here. These macronutrients constitute major components of the spermatozoa. Effects of nutritional elements on the development of Sertoli cells and Leydig cells, sperm motility and semen quality, capacity of capacitation and fertilization are discussed. A review of these areas will provide researchers with a better understanding of the compulsory participation of these nutrients in male reproductive processes. This review also pointed out gaps in current studies which will require further investigations.
\end{abstract}

Keywords: Spermatogenesis; Nutrition; Zinc; Selenium; Vitamin

\section{INTRODUCTION}

Vertebrate male reproductive endocrine systems commonly comprise hypothalamus, anterior pituitary gland and testes, which form the hypothalamic-pituitary-gonadal (HPG) system. The male reproductive tract mainly consists of testes, epididymis and ductus deferens [1,2]. The major function of the male reproductive tract is to produce spermatozoa and hormones. The anterior pituitary gland produces two hormones: follicle-stimulating hormone (FSH) and luteinizing hormone (LH) (Tables 1 and 2) [1].

Undernutrition poses adverse effect on the reproductive capacity of males. The restriction of nutrient intake or deficiency of particular nutrients in experimental animals delays sexual maturity and causes rapid regressive changes in male accessory organs. Therefore, successful reproduction requires complete provisions of macro- and micronutrients, including zinc [3], vit A (retinol) [4], vit $B_{12}$ [5], vit $B_{9}$ [5], vit $E$ [5], vit D [6], folate [7], selenium [8], nickel [9], manganese [10], chromium [11], copper [11], fatty acid [12], protein [13], arginine [14], and carnitine [15].

Table 1. Major function of male reproductive system [1].

\begin{tabular}{cc}
\hline $\begin{array}{c}\text { Male Reproductive } \\
\text { System }\end{array}$ & Functions \\
\hline 1) Hypothalamus & $\begin{array}{c}\text { Secretion of gonadotropin releasing hormone } \\
\text { (GnRH) which stimulates anterior pituitary }\end{array}$ \\
2) Anterior pituitary & $\begin{array}{c}\text { Secretion of follicle-stimulating hormone } \\
\text { (FSH) which involves in sperm ma-turation } \\
\text { and luteinizing hormone (LH) which stimu- } \\
\text { lates production of testosterone }\end{array}$ \\
3) Testes & Secretion of testosterone \\
\hline
\end{tabular}

Table 2. Main function of male reproductive tract [2].

\begin{tabular}{|c|c|}
\hline Male Reproductive Tract & Functions \\
\hline 1) Testes & Sperm production \\
\hline 2) Epididymis & $\begin{array}{l}\text { Sperm acquisition of motility, maturation } \\
\text { and Storage }\end{array}$ \\
\hline $\begin{array}{l}\text { 3) Ductus deferens, acces- } \\
\text { sory glands, penis }\end{array}$ & Sperm transportation into female tract \\
\hline
\end{tabular}




\section{WHAT ARE NECESSARY NUTRITION FACTORS FOR THE PROCESS OF SPERMATOGENESIS?}

\subsection{Functions of Several Micronutrients during Spermatogenesis}

Spermatogenesis is a precisely organized process by which normal diploid cells turn into sperm which occurs within the seminiferous tubules. Three distinct phases can be divided in the process of mammalian spermatogenesis which are: proliferation of diploid spermatogonia, meiosis of spermatocytes into haploid spermatids, and differentiation of haploid spermatids during spermiogenesis [4]. Round spermatids are differentiated into elongated spermatozoa with midpiece and tail at the final phase of spermiogenesis.

As a result of extensive chromatin remodeling and compaction during spermiogenesis, spermatozoa with highly condensed nucleus are formed [4]. Spermatozoa are released into seminiferous tubules, spermatogonia and immature spermatocytes gather at the basal compartment of seminiferous epithelium. The seminiferous tubule is the main compartment of the testes and also the site of sperm cell formation and maturation $[4,16]$. In this paper, we shall discuss about how nutrition affects the process of spermatogenesis.

\subsubsection{Functions of Zinc during Spermatogenesis}

Zinc plays several roles in the male reproductive system, one of them is its participation of ribonuclease activity which is highly active during the mitosis of spermatogonia and meiosis of spermatocytes (Table 3). Lack of zinc causes declination of ribonucleic acid (RNA), deoxyribonucleic acid (DNA), and protein level in testes of zinc-deficient rats accompanied by increasing ribonuclease activity. Hence, the role zinc plays in the process of cell growth as a cofactor for both DNA- and RNApolymerase activities and for RNA-dependent DNA polymerase is significant. A reduced rate of fertility in some species is attributed to a reduction of total RNA and protein content in spermatozoa which further emphasizes the vital function of zinc in polymerase activities [3]. Apart from RNA- and DNA-polymerase, zinc also associates with metalloenzymes like phosphatases, carbonic anhydrase, and alcohol dehydrogenase. Detailed mechanisms about how zinc affects ribonuclease activities might give a deeper insight in the importance of zinc in the initiation of spermatogenesis.

After completing mitosis and meiosis, haploid spermatids enter spermiogenesis, a process of physical and physiological maturation. During spermiogenesis, spermatids acquire tail and motility with a developed midpiece which connects the head compartment with the tail. This physical maturation process mostly happens in
Table 3. Functions of Zinc during different phases of spermatogenesis.

\begin{tabular}{cc}
\hline Spermatogenesis & Functions of Zinc \\
\hline $\begin{array}{c}\text { 1) Initiation of spermato- } \\
\text { genesis }\end{array}$ & Involves in ribonuclease activity [3]. \\
2) During spermatogene- \\
sis & $\begin{array}{c}\text { Participate in spermatozoa maturation [3] } \\
\text { Preserve germinative epithelium and } \\
\text { seminiferous Tubule [17]. }\end{array}$
\end{tabular}

3) The end of spermatogenesis (spermiogenesis)

Enhance sperm motility [3].

seminiferous tubule. Sperm maturation and storage occurs in the epididymis [16]. Epididymis also forms the blood epididymal barrier which allows the epididymis to regulate and modify the luminal fluid contents. Different epididymal fluid contents in caput epididymis, corpus epididymis and cauda epididymis changes the surface of the sperm plasma membrane into a fully matured condition and provides spermatozoa with motility and capacity for fertilization as the immotile immature spermatozoa transits through the epididymis. Therefore, it is shown that the luminal microenvironment of the epididymis is vital for successful sperm maturation [2]. Finally, after a series of morphology changes, mature spermatozoa are stored in a quiescent state. Seminal plasma is also very important in sperm nutrition and function, including activation, protection against bacteria or immune cells and preparation of spermatozoa for fertilization [18]. Therefore, the wellness of seminiferous tubule and epididymis is particularly important in assisting spermatids to undergo maturation successfully [2].

Since the concentration of zinc is so high in male sex organs like prostate, testicles and in the spermatozoa itself, its important role in reproduction is undeniable [19]. Zinc is not only involved in anatomical development and normal function of male reproductive organs, but also enhances spermatogenesis by actively participating in the maturation of spermatozoa and the preservation of germinative epithelia. Therefore, low zinc intake levels might incur delay of testicle development and cessation of spermatogenesis. Experiments by Oliveira C.E.A et al. demonstrates that animals fed with $\mathrm{ZnO}$ produce a higher spermatozoa volume than other groups [19]. On the other hand, it is demonstrated that rats fed with a zinc-deficient diet for 90 days showed a surprisingly low number of sperm and an abnormal development of seminiferous tubules almost or completely lacking of germ cells lining with Sertoli cells only [17]. Karl E. Mason and co-workers suggested that degenerative changes caused by pre-pubertal short term zinc deficiency are reversible by zinc repletion, whereas atrophy 
of testicle development cannot be repaired by repletion. In their study, the hemicastration result of zinc-deficient diet rats generally indicate immature features with varied degrees of degeneration in the germinal epithelium [17]. Yet, other researchers suggested that effects of zinc shortage act only at the testicular level and bring about spermatogenesis failure [3]. Studies showed that zinc concentration in the blood closely affect spermatogenesis because zinc shortage leads to gonadal dysfunction like a decrease of testicular weight and shrinkage of seminiferous tubules [20]. It is also reported that Zinc supplementation protects against the damage effects of $\mathrm{Pb}$ which causes degenerative changes of sperm at maturation levels [21].

While the spermatids mature into spermatozoa, concentration of zinc in the testis increases and this is probably due to the synthesis of an intracellular zinc protein complex. This phenomenon could be attributed to the increased activity of zinc-containing enzymes such as lactate dehydrogenase in the mitochondrial sheath of sperm cells. High concentration of zinc and active carbonic anhydrase activity can be also observed in the rat dorsolateral prostate [3].

Closely related with sulfhydryl groups and disulfide linkages, zinc is highly concentrated in the tail of mature spermatozoa and is involved in sperm motility by controlling energy utilization through the adenosine triphosphate system and phospholipid regulation [3]. Evidence has accumulated that zinc incorporates into the developing spermatid as spermatogenesis reaches more advanced stages, especially at the tail of fully differentiated sperm where most of the zinc are concentrated. Zinc works as a regulator of enzyme activity in the semen by mediating metabolic regulation of sperm. Concentration of zinc diminished along with sperm maturation since it has been demonstrated that spermatozoa from the testis, cauda epididymis, vas deferens, and ejaculate contained less zinc than testicular spermatozoa [3]. During maturation of sperm in the epididymis, both zinc and -SH contents decrease because sulfhydryl groups are oxidized to form -S-S crosslinks [3].

Although the consequences of zinc deficiency in sperm function are widely known, the many roles for zinc in mammalian spermatogenesis are sophisticated and rarely understood, particularly the biochemical and physiological aspect. Yet, it can be concluded that, morphologically, Zinc helps to stabilize chromatin and membrane in sperm and enhances mechanical properties of sperm such as normal flagella, midpiece formation and sperm motility [3]. Besides that, the mechanism of $\mathrm{Zn}$ in maintaining a healthy development of the germinal epithelium and seminiferous tubule remains unclear. More investigations and explorations on the physiological and biochemical mechanism of Zinc in spermatogenesis are worth to be conducted as these findings might contribute greatly to a better understanding of zinc in the male reproductive system.

\subsubsection{Functions of Selenium during Spermatogenesis}

As mitosis and meiosis happen at a high rate in the seminiferous tubule and the location of germ cells which is adjacent to the phagocytic cell type, germ cells especially spermatogonia, pachytene spermatocytes and round spermatids are potentially susceptible to free radical damage [22].

Several studies have shown the protective anti-oxidant properties of Selenium (Se). Known as an essential trace element which is fundamental to human health, Se participates in the processes of cell growth, apoptosis and modification of cell signaling systems and transcription factors [23]. Se serves as a potent antioxidant which acts with an essential component of seleno-proteins by modifying their expressions. Se incorporates with selenoamino acids, (L-selenomethionine, L-selenocysteine) and selenoenzymes, such as GPxs by replacing sulfur in proteins. Critically affecting sperm quality and male fertility is glutathione peroxidases 4 (GPXs4) [23]. Therefore, spermatozoa may be more vulnerable to oxidative stress if the Se content in selenoproteins is low and likely decreases the possibility of fertilization [23].

It has been reported that Gpx4 efficiently protect cell membranes from free radicals and is predominantly found in germ cells. Gpx4 first appears in pachytene spermatocyte stages VII-X during sperm development. Its expression progressively increases from the stage of round spermatids to elongated spermatids [23].

GPx4 is initially soluble with active peroxidase activity in spermatids. However, as the spermatids mature into spermatozoa, GPx4 turns insoluble and enzymatically inactive. This insoluble form of the protein plays a role in the condensation of chromatin by forming disulfide cross-linking in sperm maturation and male fertility. Histones in chromatin of mature spermatozoa are replaced by a linkage between thiol groups of protamines which stabilize the helicoidal form of mitochondria in the spermatozoan midpiece [24]. Since most of the Se in testis is incorporated into Gpx4, studies have proven that Gpx4 acts as a structural protein which is essential for the accurate formation of the midpiece during sperm maturation. Therefore, it is understandable why Se concentrations in rodent testes exceeds that of other organs except kidney [25]. GPx4 knockout mice contains higher levels of the protein thiol in spermatozoa which demonstrates that the stability of the sperm midpiece depends on the presence of mGPx4 (mitochondrial GPx4) and its protein thiol peroxidase activity [25]. Significant decrease in polymerized GPx4 which leads to low sperm 
counts and poor sperm quality can be observed in sperm of infertile human subjects. As the absence of GPx4 is a consistent feature in oligoasthenozoospermic patients, Schriever suggested dietary Se deficiency alone is unlikely to be the solely reason of infertility [25]. Since there are controversies on this issue, it is worth to be investigated whether Se deficiency by itself might cause a pronounced effects on sperm maturation, thereby causing infertility or it is associated with the absence of GPx4.

The removal of hydrogen peroxides and fatty acid peroxides by GPx4 is accomplished by converting reduced glutathione (GSH) to oxidized glutathione (GSSG). While, gluthathione reductase (GR) is responsible for restoring glutathione into the original reduced form [24].

$$
\begin{gathered}
\mathrm{GPx} 4 \\
2 \mathrm{GSH}+\mathrm{H}_{2} \mathrm{O}_{2} \rightarrow \mathrm{GSSG}+2 \mathrm{H}_{2} \mathrm{O} \\
\mathrm{GPX} 4 \\
2 \mathrm{GSH}+\mathrm{ROOH} \rightarrow \mathrm{GSSG}+\mathrm{ROH}+2 \mathrm{H}_{2} \mathrm{O}
\end{gathered}
$$

Hence, GPx4 is essential in protecting cells from the harmful effects of reactive oxygen species and toxic metabolites by preventing lipid peroxidation of membranes in mammalian spermatozoa [22]. Human studies also indicated that the morphological integrity, viability, and motility of sperm is closely associated with GPx4 activities [25]. Another selenoenzyme which is expressed in testis is thioredoxin reductases (TRs). Both the selenoenzymes have the capability to serve as antioxidants thereby modifying the cellular redox state of cells and thyroid hormone metabolism.

In a study where mice were fed with different levels of Se, GPx4 activity markedly decreased in the liver and testis in the Se-deficient diet group and this exposes germ cells to oxidative stress. Apart from that, the number of pachytene spermatocytes and young and mature spermatids in Se-deficient diet groups exhibits significant reductions. The oxidative stress imposed within the cell has not only reduced sperm number, but also the fertility ability of mice. Studies showed that 0.1 to 0.3 mg of Se per kilogram of diet is fairly adequate for most animals [22]. In summary, Se which helps to prevent oxidative damage is proven as an essential micronutrient during spermatogenesis.

Nutritional studies have demonstrated the critical role of Se in testis, sperm, and reproduction by showing that there is a significant increase of Se content in testis when spermatogenesis initiates [25]. The high Se concentration during spermatogenesis is related to its protective property and its associated enzymes, such as mitochondrial capsule protein in sperm.

Such increase in Se content during maturation is probably to ensure that the amount of Se is sufficient to taken into the spermatozoa [22]. Despite the many studies on the effects of Selenium during spermatogenesis, more detailed investigations are required in order to provide a more clear understanding of Selenium functions, especially during its absence.

\subsubsection{Functions of Folate during Spermatogenesis}

Well known for its significance for DNA, RNA and amino acid (cysteine, methionine) synthesis, folate is an essential micronutrient for the development of germ cells [26,27]. It is available from various kinds of food, such as green-leaf vegetables, liver, fruits and etc. Folate metabolism is critical for proper cell function, as abnormal folate metabolism pathway may lead to erroneous situation, like abnormal chromosomal segregation and DNA strand breakage. Consequently, these abnormalities negatively impact the mitosis and meiosis processes of germ cells, thereby harass the normal process of spermatogenesis. The strong antioxidant property of folic acid and its synthetic form, allows it to effectively scavenge free oxidizing radicals which is important to protect DNA from oxidative stress [27]. Researchers reported a significant increase in normal sperm count in subfertile men after they were supplemented with folic acid in combination with zinc sulphate [26]. Since folate possesses antioxidant properties and enhances sperm production, it possibly inhibits apoptosis which results from oxidative damage of DNA in spermatozoa, thereby improving male fertility. Besides protecting DNA from oxidative threat, other possible functions of folate involved during spermatogenesis are as yet not well understood.

\subsubsection{Functions of Vitamins during Spermatogenesis}

Vitamins are well known for their importance in the process of metabolism (Table 4). The influence of various vitamins on the reproductive capacity is studied by several researchers. Early histological studies have shown that vitamin deprivation in dietary intake has an impact on the male reproductive system. Vitamin $\mathrm{B}_{12}$ in its diverse forms are crucial in cell replication, particularly RNA and DNA synthesis [5]. Research also indicated that retinoic acid, the alternative metabolite form of vitamin A (retinol) controls spermatogonia differentiation and spermatid adhesion characteristics [5].

In view of histopathological abnormalities, vitamin deficiencies results in severe impairment of testicular tissues and degenerative changes. These negative alterations included atrophy of the seminiferous tubules, seminiferous tubule structure abnormalities, hypoplasia of the germinal epithelium, spermatogenic arrest and also necrosis of the germ cells [5]. The frequent occurrence of giant cells in the testis of vitamin $\mathrm{B}_{12}$ deficient diet treated mice may be the manifestation of cytokinesis failure or 
Table 4. Functions of various vitamins during spermatogenesis.

\begin{tabular}{lc}
\hline Vitamins & Functions during spermatogenesis \\
\hline 1) Vit $\mathrm{B}_{12}$ & $\begin{array}{c}\text { Involves in RNA and DNA synthesis [5] } \\
\text { Promotes healthy growth of seminiferous tubule [5] } \\
\text { 2) Vit } \mathrm{B}_{9}\end{array}$ \\
& $\begin{array}{r}\text { Promotes healthy sperm and seminiferous tubule develop- } \\
\text { ment [5] }\end{array}$ \\
3) Vit A & Spermatogonia differentiation and spermatid adhesion \\
\end{tabular}

Promotes healthy reproductive organs development [28] 4) Vit $E$ Prevents sperm cell membrane from lipid peroxidation [28] Defends sperm from ROS-related events [28]

5) Vit $C$

Protect sperm from oxidative stress [29]

germ cell group assimilation. These spermatid giant cells which contain multi nucleus are formed by fusion of impaired spermatids or continuous nuclear cleavage without cytoplasmic division during spermatocyte stages, subsequently maintaining some degree of syncytium [5]. Vitamin $\mathrm{B}_{12}$ deficiency not only brings about declination number of spermatogonia, but also defective development of sperms and spermatids, reduction of testicular weight and epididymal sperm counts in rats. On the other hand, it is reported that vitamin $\mathrm{B}_{9}$ deficiency on human and mice results in vacuolization and necrosis in the germinal epithelium of testis. Prominent lesions induced by Vit $\mathrm{B}_{9}$ deficient dietary consumption in rats include seminiferous tubules degeneration, spermatogenesis degradation and magnification of morphologically abnormal sperm [5] (Table 4).

Whereas, shortage of Vitamin E may induce reproductive organ damages, such as spermatogonium degeneration, testicular dysfunction and seminiferous tubules shrinkage. Many studies have shown remarkable effects of Vit E which works against oxidative stress in various animal organs. Vitamin $\mathrm{E}$ is also important in the healthy maintenance of male reproductive organs and the survival of spermatids. The role of Vitamin $\mathrm{E}$ in the reproductive system is widely studied and it has been demonstrated that Vit E enhances the development of reproductive organs by increasing epididymal weight, epididymis ductules and seminiferous tubules diameters, spermatogenic cells and interstiticial cell density which is crucial in the smooth-going of spermatogenesis [28].

Vitamin E consists of a group of lipid soluble compounds, tocopherols and tocotrienols, is one of the most important antioxidative molecules in defending oxidative stress and preventing the production of lipid peroxide by scavenging free radicals (Table 4). Besides that, antioxidant activities of superoxide dismutase (SOD) and glutathione peroxidase (GSH-PX) intesticular cell membrane and mitochondria are promoted [28]. As a result of spinal cord injury (SCI), the reactive oxygen species (ROS)-related events become extraordinarily active and consequently cause male infertility such as poor sperm motility and abnormal sperm morphologies and low sperm counts. Shulun Wang et al. reported the protective effects of Vitamin $\mathrm{E}$ in sperm functions of spinal cord injured rats [28]. The lack of Vit $\mathrm{E}$ can, therefore, cause testicular abnormalities, defective spermatogenesis, faulty differentiation of epididymal epithelial cells, incomplete spermatogenesis and spermatid degeneration.

Another type of vitamin which is also important in normal functioning of the male reproductive system is Vitamin C, namely ascorbic acid. This water-soluble vitamin has been reported to be associated with fertility as it is very concentrated in the epididymal fluid and seminal plasma. Vitamin C intake closely associates with sperm numbers, concentration and motility. Its protective role within the epididymis is manifested through its reductive property which prevents sperm from oxidative impairment of DNA, thereby maintaining the genetic integrity of sperm [29].

Exogenous supplementation with ascorbic acid brings a marked increase in the concentration of ascorbic acid in the testes and blood plasma, while a significant reduction level of lipid peroxidation is observed in these locations. Normally, ascorbic acid levels in healthy adults range from 1.9 to $12 \mathrm{mg} / \mathrm{g}$. Results of male rats supplemented with 500, 250 and 0 (control) $\mathrm{mg} / \mathrm{kg} /$ day of ascorbic acid for 8 weeks, respectively, demonstrated that ascorbic acid ameliorates male reproductive traits which links with a successful high fertility rate. In a research using a combination therapy of $400 \mathrm{mg}$ of GSH, $200 \mathrm{mg}$ of vitamin $\mathrm{C}$ and $200 \mathrm{mg}$ of vitamin $\mathrm{E}$ for 2 months, sperm concentration is markedly improved and the level of oxidative DNA damage in the spermatozoa is reduced [27].

The beneficial effects of vitamins for a suitable development of the male reproductive system are widely studied and are mostly emphasized with respect to their oxidative resistance. It remains unknown whether excessive vitamin dietary intake may bring deleterious effect to sperm and testicular function. Besides that, the molecular mechanisms of vitamins in improving spermatogenesis and testicular development are yet little understood. Further investigations on mechanism of vitamins in enhancing spermatogenesis may assist in curing infertility.

\subsubsection{Functions of Nickel and Manganese during Spermatogenesis}

Other trace metals which also play a minor role in spermatogenesis include nickel (Ni) [9] and manganese (Mn) 
[10]. It is reported in earlier studies that nickel deprivation causes defects in reproductive function. It is suggested that nickel influence cyclic nucleotide gated (CNG) channels which can be found in the reproductive system by sensitizing cyclic guanosine monophosphate (cGMP)-gated cation channels or vice versa [9]. The hypothesis that nickel affects CNG channels is supported by other studies. Nickel deficiency diminishes the sperm production in testis, sperm counts in the epididymis, efficiency of spermatozoa epididymal transit, and sperm motility. These effects might be also related to cGMP action. The balance of nickel level in the body is important to maintain a normal reproductive performance as both deficient and excessive intakes of nickel might provide negative effects. The consequences of excessive nickel consumption in male rats include increased testicular nickel, atrophy of seminiferous tubules, decreased spermatids, and diminished fertility [9]. However, the accurate concentration of nickel which is beneficial or adverse to male reproductive performance is not mentioned in this study. In addition, administration of $\mathrm{MnCl}_{2}$ at $25 \mathrm{mg} / \mathrm{kg}$ dose accelerated spermatogenesis as a significant increase of daily sperm production and efficiency of spermatogenesis can be observed in the experiment. Even though $\mathrm{Mn}$ is suggested as a stimulator of pubertal development, this metal may cause male precocious pubertal development if exposed to moderately elevated levels of Mn too early in life [10]. More particular research about the effects of nickel and manganese or even other not-mentioned minerals on spermatogenesis is needed as relevant studies are scarcely found.

\subsection{Functions of Various Nutritional Elements on Sertoli Cells and Leydig Cells}

Sertoli cells, namely nurse cell lines with the inner walls of the seminiferous tubules and participate partly in the process of differentiation by providing structural and nutritional support for developing germ cells. The materials secreted by Sertoli cells provide transportation for non-motile spermatozoa from testes into the efferent duct system. There are different spermatogenic cell types in seminiferous tubules as the timing of spermatogenesis is under rigid control, this phenomenon is known as the seminiferous epithelial cycle [1,2]. Besides Sertoli cells, the cell type that is interspersed within the seminiferous tubule, the so-called interstitial cells, are known as Leydig cells. Leydig cells which produce the male sex hormone, testosterone are the major sites of steriodogenesis in testes also play a crucial role in sperm maturation [2].

As a consequence of vitamin deficiencies, intertubular tissues manifest distinct degrees of degeneration of the insterstitial Leydig cells. Yet, Sertoli cells which are cru- cial in the division of spermatogonia differentiation of the treated groups did not exhibit any abnormalities and were apparently not affected by vitamin insufficiency. This proved that resistance of Sertoli cells to vitamin deficiency is obviously stronger than germ cells [5]. It is also reported that zinc deficiencies in men can injure angiotensin-converting enzyme activity in Leydig cells which consequently lead to testosterone depression and the impediment of spermatogenesis [20].

Most nutritional studies on the male reproductive system emphasize on testicular development and spermatogenesis, while Sertoli cells and Leydig cells were not studied in detail as yet. Further investigations about nutritional effects on the development of Sertoli cells and Leydig cells are hence needed since relevant studies are scarce.

\subsection{Functions of Various Nutritional Elements on Sperm Motility}

During spermiogenesis, the potential for forward motility starts as the spermatozoa pass through the epididymis, while the capacity for spermatozoa to move forward is acquired in different regions of that organ. At the beginning of epididymal maturation, the spermatozoa in epididymidis caput are immotile. As the spermatozoa travels from the caput of the caudal epididymidis the membrane of the spermatozoon tail obtains new properties, the undulating movements of spermatozoa alter gradually [2]. Besides factors from the caudal epididymal plasma, the induction of forward motility requires also an increased cyclic AMP level and calcium ion. During epididymal transit, the intrasperm level of cAMP and the ATPase activity increases which is essential for the induction of sperm motility. A specific glycoprotein which involves the induction of forward movement binds to the spermatozoa and it is also present in the testicular, epididymal and seminal fluids. Even though the presence of calcium ions is not always necessary depending on species, calcium ion is crucial for the initiation of sperm motility. It is reported that sperm motility is proportional to the free calcium level found in the sperm of the caudal epididymis [30]. Trace minerals and vitamins also influence sperm motility.

Zinc participates in enhancing sperm motility by adenosine triphosphate system and phospholipid regulation, being highly concentrated in the tail of mature spermatozoa [3] (Table 5). Interestingly, even though zinc content is directly proportional to sperm quality, it is negatively correlated with sperm motility and velocity. Our findings show that zinc content which is high in immobile starfish spermatozoa diminished significantly after sperm swims vigorously. This suggests that zinc or 
Table 5. The functions of various nutritional elements on sperm motility.

\begin{tabular}{cc}
\hline $\begin{array}{c}\text { Nutritional ele- } \\
\text { ments }\end{array}$ & Functions in sperm motility \\
\hline Calcium & Initiates sperm motility [30]. \\
Zinc & $\begin{array}{c}\text { Enhance sperm motility through ATP system and } \\
\text { phospholipid regulation [3]. }\end{array}$ \\
Selenium & Assure the accurate formation of midpiece and fla- \\
gella [31,32]. \\
Nitamin E & Improve mitochondrial function [6]. \\
Change CNG cation channel function [9].
\end{tabular}

zinc-containing compounds have left sperm and dissipate to the external environment. This is probably achieved by the liberation of zinc to histidine or other metal-chelating from the acrosome, the catalytic activity affects the physical state of spermatozoa and thereby stimulates its activation [3]. Zinc participation in sperm motility can possibly be explained from the perspective of ATP system mechanism which involve fibrillar contraction in the axial filament and utilization of energy reserves for the sperm. It is suggested that zinc which is localized in the sperm midpiece together with a lipoprotein fraction liberates free phospholipids simultaneously when zinc is released from sperm, this catabolism of lipid consequently contributes energy required for movement of spermatozoa. The modification of membrane structure by zinc increases sperm activation [3].

Early studies reported that Se-deficient rats were sterile, lacking well-developed immotile sperm which showed an irregular structure of mitochondria, such as swollen mitochondria, bending between midpiece and tail, structural abnormalities between head and midpiece [31]. Mitochondrial function is especially important for the optimum capacity of sperm motility as mitochondria are highly concentrated at the tail. It is common that flagella defects occurred primarily at the midpiece which impair sperm motility is constantly observed in Se-deficient animals [23]. Due to the deprivation of Selenium, the functional activity of the sperm glutathione peroxidase and/or the thioredoxin system is compromised which in turn results in oxidative damage and abnormal mitochondrial remodeling. The flagella abnormalities include both hairpin-like bends of the flagellum, breakage of its axial fiber, premature termination of the mitochondrial sheath and others. A gap in the posterior midpiece is exhibited in late spermatids and testicular spermatozoa which reflects the disappearance of the mitochondrial sheath [32]. Research proved the importance of Se in the formation of the midpiece by showing that labeled Se injected into Se-deficient rats was retained in the testis and concentrated in the midpiece of sperm [25].

Se supplementation such as SeMet and selenocysteine is reported for its ability to enhace sperm motility [33]. Yet, another study demonstrated a totally different result where sperm quality and fertility of specimens ameliorated after Se supplementation [23]. Even though Se supplementation brings positive effects to sperm production, excessive selenium diet may lead to reduced sperm motility (Table 5). In an experiment where eleven healthy men were fed with different levels of Se diets, the number of motile sperm in the high-selenium group decreased and ended up at $18 \%$ lower than at the beginning [8]. The detailed molecular mechanism which causes high levels of immotile sperm by excessive Selenium remains unknown as yet. Further investigations to probe this question are required in order to understand how Selenium uptake disrupts spermatogenesis.

The Vitamin E treatment group has displayed preserved sperm viability and improved mitochondrial function which counterbalances the negative effects of free radicals [28]. In addition, the ameliorative effect of Vit $\mathrm{E}$ is also approved by N. J. Chinoy et al. in a study of fluoride toxicity on reproductive functions of male mice [6]. Fluoride is toxic to normal protein metabolism, glycolysis, and the respiration process. A marked decrease of protein levels in caput and cauda epididymides and vas deferens is likely to cause incomplete acrosomal membrane structures has been demonstrated after 30 days of $\mathrm{NaF}$ treatment. Therefore, it can bring deleterious effects to sperm motility and function. The results of Vit E supplementation on $\mathrm{NaF}$ treated mice indicated that Vit $\mathrm{E}$ is capable of recovering all $\mathrm{NaF}$ induced effects on reproductive functions and fertility. It is suggested that Vit $\mathrm{E}$ ameliorates sperm function by reducing cell-damaging free radical oxygen species at the cellular-molecular level [6]. However, the detailed mechanism about how Vit E acts in improving sperm motility is yet unclear. Human studies with asthenospermia treated by oral administration proved the protective role of vitamin $\mathrm{E}$ by improving sperm motility and impregnating their spouse. A great reduction in the MDA concentration in spermatozoa towards normospermic levels after vitamin $\mathrm{E}$ treatment therapy is a positive indicator for infertile patients [34]. A combination of SeMet and SeMet + Vit-E is reported to protect sperm from oxidative damage induced by ammonia and improve sperm motility, subsequently increasing the rate of acrosome reaction [33]. The second combination had a better ameliorative effect in enhancing sperm quality. So far, most of the studies on nutritional effects on male reproductive efficiencies apply a single type of nutrition instead of combinations of different trace minerals and macronutrients. Further studies with various combination trials are encouraged and may contribute in discovering innovative therapies in curing male infertility. 
As for minor trace metals involved in spermatogenesis, it is likely that nickel affects sperm motility by changing CNG cation channel function. This hypothesis is expound and proven by several studies of different animal species which demonstrated that sperm motility involves CGMP and CNG channels. cGMP along with soluble guanylate cyclase, nitric oxide synthase, and guanylate cyclase B are also present in the lamina propria of the semiferous tubule, and Sertoli cells, Leydig cells, and blood vessels in human testes [9]. Although nickel is believed to be important in enhancing sperm motility, morphological, biochemical and physiological significance of nickel involvement in spermatogenesis is little understood.

\subsection{Nutritional Effects on Capacitation and Fertilization}

After ejaculated into the female genital tract, spermatozoa need to get activated before they obtain the ability to fertilize an oocyte. This activation process is known as capacitation. The ultimate control of capacitation lies within the sperm plasma membrane. As the outer surface of the plasma membrane, the glycocalyx undergoes several biochemical changes, a derepression of a set of preprogrammed cellular events takes place and hyperactivates the spermatozoa. These modifications include alterations in protein and lipid composition. The microenvironment of the oviduct where bicarbonate levels are much higher than in the epididymis permits capacitation. The capacitation of sperm not only requires electrolytes such as $\mathrm{HCO}_{3}^{-}$, but also metabolic energy sources like $\mathrm{Ca}^{2+}$ and protein sources like bovine serum albumin (BSA). It is reported that loss of cholesterol promotes capacitation [35].

In order to accomplish fertilization accurately and efficiently, it is crucial for sperm to be progressive and membranes to be intact. Fertilization will be impaired if either of the two conditions are not fulfilled. Peroxidative damage to the sperm plasma causes membranes to loose their fluidity and integrity, which consequently impairs the competence of spermatozoa to involve in membrane fusion events, like the acrosome reaction linked with fertilization. As a result of exposure to high levels of oxidative stress, fertility of spermatozoa is significantly affected, such as by low sperm motility [36].

In a study with treatment of porcine spermatozoa with Se and Vit-E, motility, viability and acrosome reaction is improved [33]. They also found that incorporation of ${ }^{14} \mathrm{C}(\mathrm{U})$-glucose is positively correlated with rates of motility, viability and acrosome reaction. Combination of glucose and other monosaccharides is the major exogenous energy source to promote an acrosome reaction in vitro [22]. Reduced fertility is also observed in Vit D deficient male rats. After administration of vitamin D and its active metabolite, 1,25-dihydroxycholecalciferol, fertility is restored from a 0.11 mating ratio to 0.24 and 0.22 respectively. Yet, Ann M. Uhland et al. suggested that the influence of vitamin $\mathrm{D}$ and its active metabolite, 1,25-dihydroxycholecalciferol, on male fertility is indirect as they act on classic target tissues and regulate levels of calcium in reproductive tissues [37]. While zinc which has an inhibitory effect on capacitation plays an important role in decapacitating sperm during the spermatozoa-ZP interaction, particularly ZP-induced acrosome reaction by binding to the sperm plasma membrane [3]. Although nickel is believed to be important in enhancing sperm motility, morphological, biochemical and physiological significance of nickel involvement in spermatogenesis is little understood.

Studies on nutritional elements which promote high quality capacitation and fertilization are scarcely found. More investigations on this aspect are urgently needed as the results may contribute greatly in curing male infertility.

\subsection{Nutritional Effects on Sperm Quality and Semen Quality}

Various studies indicated that sperm quality is enhanced by zinc, selenium, folate and vitamin supplementation. Sperm quality was ameliorated after replenishment of zinc in infertile patients [21]. Whereas, diminished Se concentration is linked with low sperm production and poor sperm quality. In a research about paternal nutrition on aneuploidy in sperm, it is demonstrated that frequencies of sperm with disomies X, 21, sex nullisomy, and a lower aggregate measure of sperm aneuploidy are much lower in men with high folate intake than men with lower intake. In the study, consumption of $400 \mathrm{mg}$ to $700 \mathrm{mg}$ total folate per day is adequate to reduce the rate of sperm aneuploidy [27]. Well known of the importance of vitamins in metabolism process, ascorbic acid replenishment (more than $200 \mathrm{mg}$ /day) lead to improved sperm quality in heavy smokers [38]. Adequate diet without vitamins demonstrates a marked diminish of testis weight and male sex hormones in experimental animals which negatively influence sperm quality [5].

Sperm quality and quantity is also closely associated with semen quality. The various contents in semen play a significant role in sperm survival and semen quality is a parameter of male fertility $[17,39]$. Semen is formed during the process of ejaculation, when sperms pass through the ejaculatory ducts and combine with fluids secreted by seminal vesicles, prostate and bulbourethral glands. Fructose which is secreted from the seminal vesicle is especially rich in semen because it provides nutrient energy for the spermatozoa. Seminal plasma is vital because it provides a nutritive and protective envi- 
ronment for spermatozoa to survive, particularly during their journey through the female reproductive tract that is hostile [40].

Commonly, most seminal plasma and spermatozoa in mammals are highly rich in zinc, 30 times higher than zinc concentration in blood. Yet, it varies among different mammalian species. Seminal plasma zinc originates from the prostate [20]. The relationship between zinc concentration in seminal fluid and sperm quality is discussed here in order to understand the effect of zinc in seminal plasma.

Studies demonstrated that adding zinc to washed human spermatozoa counteracts succinate which directly induces the enhancement of sperm respiration activity. High spermatozoa respiration activity provides an indication of cellular and metabolic disorganization. Therefore, the role of zinc for the integrity of spermatozoa is obviously significant [3]. Zinc in semen which binds to the sperm plasma membrane after ejaculation possibly further stabilizes sperm nuclear chromatin DNA during sperm transportation in the female reproductive tract. The antioxidant property of zinc also helps to counteract reactive oxygen species produced by the spermatozoa which may affect nuclear chromatin stability and sperm motility [3,17].

However, several in vitro studies have reported that zinc and seminal plasma (SP) pose inhibitory effects on sperm capacitation including hyperactivation and acrosome reaction, and also spermatozoa-zona pellucida binding and penetration. A related review has pointed out that spermatozoa in seminal plasma with high zinc concentration and low phosphatase activity shows a high percentage of dead and immobile spermatozoa. Likewise, high zinc concentration $\left(1 \mathrm{mmol} \cdot \mathrm{L}^{-1}\right)$ in the culture medium of spermatozoa from fertile men in an in vitro study results in both sperm motility and sperm penetration dysfunction of ZP-free hamster oocytes [20]. Thus, it is common that studies showed that concentration of zinc in human sperm cells is almost 33 times as high as that of seminal plasma. Yet, the study showed that there is no relation between zinc concentration in spermatozoa and sperm density, progressive motility, oxygen consumption, or fructose composition in plasma [3].

Many studies have shown that seminal zinc concentration positively correlates with sperm count and duration of abstinence, but inversely correlates with semen volume. High seminal zinc concentration in men with normozoospermic semen may result in capacitation failure during zona pellucida (ZP) binding and the ZP-induced acrosome reaction (ZPIAR) [20]. As mentioned before, high SP zinc level causes adverse effects on sperm motility.

Diverse conclusions were expressed on whether high seminal plasma zinc concentration affects sperm quality positively, negatively or shows no effect at all. While some studies showed low zinc level in semen, most studies have proven that semen and its constituents contain relatively high concentrations of zinc. Further investigation of the distribution of zinc in semen and its relevant impact on sperm quality is needed to resolve this dispute. Besides, more studies on the physiological mechanism and implications of seminal plasma zinc in sperm function are required in order to understand the inhibitory or enhancement effect of zinc. Perhaps, other prostatic secretions (e.g. citric acid) might also influent zinc concentration and sperm function.

Selenium can also be found in semen and has an important effect on semen and sperm quality. Some research suggests that sperm count and concentration of Selenium in semen is significantly in direct ratio. Bleau et al. points out that sperm motility reach its optimal performance within the range of 40 to $70 \mathrm{ng} / \mathrm{ml}$ semen Se levels in men [41]. In contrast, above and below this range, significant decrease of sperm motility and remarkable increase of asthenospermia rate is observed in men [41].

Besides fructose, human semen consists of high levels of calcium (Ca), magnesium (Mg), and copper (Cu) [18]. They are bound in ionic form. It is pointed out that abnormal levels of these elements may affect spermatogenesis with respect of sperm production, maturation, motility, and fertilizing capacity [18]. In a research about effects of multiple trace metals on semen quality, $\mathrm{Mg}$ in seminal plasma significantly affects sperm concentration, but not motility. However, the authors found that calcium has neither beneficial nor detrimental effects between calcium and sperm motility. This result contradicts earlier reports that low calcium ion concentration in semen decrease sperm motility but improve fertilization ability. Besides, the study exhibited a weak but positive correlation between blood $\mathrm{Cu}$ concentrations and sperm motility [18]. Copper concentration within normal physiologic range is essential in enzymatic activities, yet high level of copper is detrimental to sperm morphology. They also found due to a positive correlation between $\mathrm{Mg}$ and Ca that Mg serves as a physiological Ca antagonist [18]. However, the mechanism of magnesium in spermatozoa quality is as yet not well understood. Results from effects of $\mathrm{Mg}$, $\mathrm{Ca}$, and $\mathrm{Cu}$ in seminal plasma are controversial, even though there is substantial evidence that these elements are involved in sperm motility. There are further studies required to resolve this question.

Several investigations tried to elucidate the importance of natural antioxidants, such as vitamins A and E in improving semen quality and sperm production through the protection of spermatozoa membranes, but a study of 
boar sperm and supplementation with vitamins $\left(\mathrm{B}_{2}, \mathrm{~B}_{6}\right.$, $B_{9}, B_{12}$, and $E$ ) showed reverse results. Most of the water-soluble vitamins seem to be transferred from the blood to the seminal plasma, except for Vit $\mathrm{B}_{6}$, while Vit $E$ is not transfered directly to seminal plasma. In spite of ameliorated effects on ejaculate volume, administration of fat- and water-soluble vitamins did not positively affect sperm production or semen quality [42]. Nevertheless, an experiment of selenium and vitamin E diet supplementary in boar showed improvement in sperm quality [33]. Moreover, supplemental Vit E at a concentration of $200 \mathrm{IU}$ per sheep a day increased semen quality and quantity and reduced the malondialdehyde (MDA) level significantly [28]. Research studies indicate that testes and seminal plasma are sensitive to the diminution of ascorbic acid which might lead to reproductive dysfunction [38]. Activities of antioxidant enzymes such as superoxide dismutase (SOD), catalase, glutathione reductase and glutathione peroxidase drop with the declination of ascorbic acid concentration of human semen, while levels of hydrogen peroxide and lipid peroxidation increase instead. This demonstrates the strong protective role of ascorbic acid in maintaining normal sperm development. However, the mechanism of ascorbic acid on antioxidant enzymes yet remains an unsolved question. Deterioration of semen quality subsequently leads to low sperm number and the increment of abnormal and dead sperm is attributed to vitamin $B_{12}$ deficiency [29].

It is worth to notice that vitamin $\mathrm{E}$ well known with its health beneficial anti-oxidant property might bring harmful impact if taken excessively. According to Miller's study, he suggests that daily doses of $400 \mathrm{IU}$ vitamin E or more might increase the risk of dying prematurely and also cause other possible serious side effects [43]. It is probably due to consumption of high dosage vitamin $\mathrm{E}$ can interfere the normal physiological ROS system in the body. Miller reported a significant increase of mortality rate at amounts above 200 IU per day and subjects whose vitamin E level was lower than 400 IU per day were less $10 \%$ likely to die than those who exceeded the amount. Whereas the risk increase more than $20 \%$ at mega doses, such as 2000 IU of vitamin E. Vitamin E doses intake is not yet regulated by the FDA or other agencies, but he recommends 400 IU per day as the "upper tolerable limit” for vitamin E [43].

Gliozzi's study on the effects of fish oil and vitamin E dietary supplementation on semen quality, sperm susceptibility to lipid peroxidation, tocopherols content and fatty acid profiles in rabbits has demonstrated the negative implications of feeding high dosage of vitamin E. Despite the zero negative result of highly concentrated fish oil intake on sperm lipid composition, an unexpected remarkable reduction of total PUFA proportion in sperm lipid composition is observed in the study [44]. While Bolle and others describe vitamin $\mathrm{E}$ as a double-edged sword, its effect either positive or negative, depends largely on its dosage. Vitamin E possesses beneficial properties in the male reproductive physiology because it prevents polyunsaturated fatty acid chain-breaking reaction in the membranes of germ cells and spermatozoa. However, certain concentration of ROS is needed in many organs, specially the reproductive organs as they are critical in regulating the onset of hyperactivation, capacitation of spermatozoa and acrosome reaction. Thus, excessive antioxidants may cause impaired sperm function by inhibiting ROS [45]. Yet, the minimum amount of ROS required for normal physiological function and without suppressing physiological concentrations of ROS remains unknown. Further understanding of the efficacy of different vitamin $\mathrm{E}$ concentrations in improving or impairing male fertility and underlying physiopathological mechanisms for effects of vitamin $\mathrm{E}$ on sperm functions and male accessory glands should be investigated. These investigation results will provide a more credible reference for further therapeutic method to enhance sperm function, and ultimately fertility.

Furthermore, thiol concentrations are found to be more abundant in the ejaculate than those in spermatozoa. This indicates the important antioxidant function of seminal plasma in protecting spermatozoa from ROS damage. Spermatozoa of fertile men have higher thiol concentrations than men with male factor subfertility (MFS) as the high GSH concentrations helps to improve sperm motility and reduce sperm morphological abnormalities by participating in the protection of cells against oxidative stress. Therefore, GSH concentration in seminal plasma provides an indication of sperm vitality (motility and intact morphology). On the other hand, it is reported that thiol concentrations in spermatozoa of asthenozoospermic men is much higher than those of normozoospermic men and this may be due to the contribution of excessive ROS level in spermatozoa which lead to the up-regulation of thiol synthesis for protection purpose [27]. The excessively high thiol concentrations subsequently reduce spermatozoan motility as the disulphide bonding during sperm maturation in the epididymis is reduced.

Several other aspects of sperm protection and sperm nutrition are not well studied as yet. There are also several controversies concerning the effects of nutritional elements in sperm plasma. Further research is required to solve the disagreements and the results may bring everlasting contributions in preserving sperm in vitro and increasing the fertility rate of sperm under lab conditions. 


\subsection{The Effects of Different Nutritional Uptake Levels on Male Reproductive Function}

Nutrition is a major factor in the effectiveness of reproductive function. It may affect the efficiency of related hormone production and the growth of reproductive organs. Studies have demonstrated that luteinizing hormone ( $\mathrm{LH})$ secretion during early gonadotropin rise is elevated and can be sustained for a longer period when calves are fed with improved nutrition during calfhood. As a result, testosterone concentration also increased associated with higher circulating insulin-like growth factor-I (IGF-I) concentrations. This consequently contributes to greater testicular proliferation and sperm production in mature bulls. As insulin-like growth factor-I receptors can be found in Leydig cells, IGF-I has great influence in regulating Leydig cell number and differentiation [46]. The decreased content of fructose and citric acid in seminal vesicles reflects the negative effect of restricted diet intake of gonadotropic hormone secretion. If gonadotropic hormone is injected, both fructose and citric acid promptly appear in the semen [47].

In experiments related to effects of nutrient deficiencies on reproductive functions, results have indicated that even though adequately fed after puberty, testicular growth which was retarded in undernourished bulls during the pre-pubertal period is irreversible [46]. Testes measurements are proportional to sperm production, therefore, reduction of testes size means a lower sperm number (Almeida, Schwalbach et al. 2007). Not only testicular development is impaired, but also testosterone level in both spermatic vein blood and testicular tissue is diminished which illustrates the significance of adequate nourishment during calfhood [47]. There is also a marked degradation on two accessory male reproductive organs, which are the seminal vesicles and Cowper's glands. The chemical changes in the secreted substance from these glandsdecreased output of fructose and citric acid is the consequence of histological changes in the seminal vesicles [47].

In addition, delayed appearance of motile sperm in the ejaculate, retarded testicular growth, decreased size of Leydig cells and low differentiation of the seminiferous tubules and of the interstitial tissue is observed in the male gonads of underfed maturing bull-calves [47]. In another study which discussed the detrimental effects of winter yield hay feeding in young boar demonstrated high frequency of sperm cell abnormalities, decreased testicular volume and scrotal circumference, once again emphasizing the essentiality of a well-balanced diet to achieve optimum reproductive efficiency in young animals [48].

The differentiation of the Leydig cells in nutrition adequacy animals is highly advanced with little dark, almost pyknotic nuclei intermingling together. Besides, the interstitial tissues observed in well fed animals are loose which is not observed in low nutrient intake animals. On the other hand, there are only a few Leydig cells in nutritional deprivation animals, most of them are in spindle shape or in transitional stages. Restricted diets significantly decrease the number of cells of the spermatogenic series at all stages and the number of Sertoli cells. Thus, it is apparent that the cellularity of the tubular epithelium is significantly lower in low nutrition animals compared to those of high nutrition [47].

These studies provide an overall insight of excessive and deficient nutrient intake in the male reproductive system and sperm development and provide a foundation for more detailed nutritional studies.

\subsection{Macronutrients Which Affect Spermatogenesis and Sperm Function}

\subsubsection{Lipid}

Fats facilitate the take-up of crucial elements for our diet and serve as sources of energy and carriers of fat-soluble vitamins. Fats also supply essential fatty acids that are vital constituents of cell membranes, and precursors of hormone-like compounds like prostaglandins. It is well known that testes contain a high content of $\mathrm{C}_{20}$ and $\mathrm{C}_{22}$ carbon atoms of polyunsaturated fatty acids (PUFAs), especially in mammalian sperm [49]. As spermatozoa are rich in lipids with phospholipids occupying approximately $70 \%$ of the total, lipids have an important role in maintaining sperm cell viability, maturity, fertility and function [50]. Phospholipids and cholesterol are major components which form the lipid bilayer of cell membranes [51]. The normal structure of the sperm membrane is crucial for successful fertilization as both the acrosome reaction and sperm-oocyte fusion are membrane-associated events [50]. Therefore, alteration of polyunsaturated fatty acid composition in sperm could have an adverse effect on its biochemical properties and functional characteristics. As the sperm membrane constitutes of a high content of unsaturated fatty acids and a lack of cytoplasmic enzymes which protects it from the attack of reactive oxygen species, spermatozoa are very vulnerable to peroxidative damage which might impair membrane fluidity [50].

In most mammals, docosahexaenoic acid (DHA, 22: $6 n-3$ ) is the major polyunsaturated fatty acid (more than $60 \%$ ). There are particular cases in other species that docosapentaenoic acid (DPA, 22:5n-6) constitutes the dominant part of sperm cell membranes. Research showed that the amount of docosahexaenoic acid in spermatozoa is proportional to sperm motility as a main portion of DHA in sperm is concentrated in the sperm tail [52]. Since sperm cells are susceptible to free radical damage, 
defense mechanisms in sperm cells are important to neutralize the toxic intermediates. MDA production, the by-products of lipid peroxidation, can be used to monitor the destruction level of peroxidation in spermatozoa. Previous studies indicated that high MDA levels are concurrent with high SOD activity in human and boar spermatozoa. SOD is the major enzymatic defense against lipid peroxidation and contributes to the prevention of peroxidative damages by dismutating the super-oxide anion radical into hydrogen peroxide [49].

PUFAs can not be synthesized endogenously from saturated or monounsaturated fatty acids and must be acquired from precursor PUFAs in diet. It is well established that dietary PUFAs can be transfer to sperm and has been shown to be effective in numerous species, including humans, fowl, boars, and rams. Findings indicated that PUFA supplementation stimulates SOD activity which is related to high sperm viability [49]. Furthermore, authors reported that decreased levels of DHA and PUFA, and ratio of n-3 to n-6 fatty acids in spermatozoa may be closely associated with infertility in oligo- and/or astheno-zoospermic men [12]. Compared to normozoospermic men, the level of DHA in seminal plasma and the ratio of omega- 3 to omega- 6 fatty acids in sperm of asthenozoospermic men is much lower. This might be the result of an excessive breakdown of PUFA induced by a high level of reactive oxygen species (ROS) [50]. It is also suggested that a high concentration of DHA in both ejaculate and spermatozoa has a positive influence on membrane fluidity which is necessary for sperm motility in humans [52]. Since various findings have demonstrated the ameliorative characteristics of PUFAs supplementation on normal sperm structure, production and function, it can be speculated that PUFAs dietary supplementation might provide a suitable therapeutic nutrition in treating male infertility.

Research findings suggested that deficiency of essential fatty acids in male rats induce testis degeneration and infertility. Supplementation of precursors to omega3 fatty acids, like cod liver oil or flaxseed oil to stallion has shown an increase of the overall level of omega-3 fatty acids in semen, but did not result in the improvement of semen quality [53]. However, experimental results using boars resulted in a higher DHA to DPA ratio in semen together with an improvement of sperm number, sperm concentration, sperm motility, sperm morphology and sperm viability [53]. Provision of tuna oil exhibited an increase of DHA and a diminution of DPA in sperm phospholipid fatty acids in human sperm after 5 weeks. The supplementation of tuna oil not only improved sperm progressive motility and normal acrosome scores, but also reduced abnormal morphological spermatozoa. DHA is crucial in promoting optimal fertility especially in human spermatozoa as it shows significant influence on sperm concentration, motility and normal morphology [51]. In contrast, in another research about the effect of DHA supplementation on sperm motility in asthenozoospermic males, results indicated that even though DHA levels increase in serum and seminal plasma, DHA supplementation had no positive influence on sperm motility [49].

\subsubsection{Amino Acid}

In a review of the role of Arginine (Arg) metabolism and nutrition, comparative evidence shows that Arg plays a crucial role as a nutritionally essential amino acid in spermatogenesis and also other important metabolic processes. It is a biochemical precursor in the synthesis of putrescine, spermidine and spermine, which are essential for sperm motility. Arg can be obtained from seafood, watermelon juice, nuts, meats, soy proteins and others. Administration of Arg has beneficial effect in treating infertility. Arg-HCl (0.5g/day) provision to infertile men for 2 months has shown significant increase of sperm counts and motility in most human specimens and achieved successful pregnancies. Similar results are also obtained from the research on boars. It is suggested that the improvement of reproduction capacity is due to the increased synthesis of polyamines and Arg-rich basic proteins in sperm cells and the regulatory role of Arg for sperm motility and capacitation [14].

L-carnitine (L-3-hydroxy-4-N,N,N-trimethylaminobutyrate) and its acyl derivatives L-acetyl-carnitine (LAC) has long been well-known as a conditionally essential amino acid for its physiologic role in health and disease. LAC provides a temporary form of transferred acetyl group and can be considered as an alternative product of LC. Both are involved in energy metabolism which promotes sperm motility, maturation and the spermatogenic process by supplying readily available energy to spermatozoa. Carnitine is assumed as a non-essential protein in human diet as it can be biosynthesized in vitro through hepatic methylation from dietary amino acids [15]. The exogenous source of LC in the diet is meat, dairy products and fish. In contrast to blood, high concentrations of LC and LAC can be found in epididymal fluids and in spermatozoa. During transit from the caput to the cauda region of the epididymis, spermatozoa which are originally immotile gradually attain the capacity of flagellar motion and accumulate high concentrations of free LC. Several reports have demonstrated that the proportion of progressive sperm motility is parallel with the concentration of free LC and LAC. Yet, the authors believe that the initiation of sperm motility is independent of the carnitine system [54]. Besides this, LC contributes to cellular energy production by accelerating the lipid metabolism, and are especially critical in mitochondrial 
$\beta$-oxidation of long-chain fatty acids [55]. In an experiment of bovine epididymal spermatozoa incubated in the absence of substrates but applying acetyl-L-carnitine as an endogenous source stimulated by caffeine, LAC is demonstrated to replace energy storage function of high energy phosphate compounds in mammalian spermatozoa [54]. Carnitine is another form of antioxidant which serves to protect cell membrane and DNA against ROSinduced damage. Research findings have also indicated that supplementation with carnitine improves sperm quality and/or quantity in testes of mice exposed to physical damage, such as heat and $\mathrm{X}$-irradiation, and in men with idiopathic oligoasthenospermia. The mature spermatozoa are protected by carnitine by the uptake of excess acetyl-CoA from the mitochondria and storing this in the form of acetyl-L-carnitine [54]. In addition, it also inhibits protein oxidation and lactate oxidative damage by removing excess intracellular toxic acetyl-CoA. L-carnitine (LC) and its acyl derivative L-acetyl-carnitine (LAC) are reported to ameliorate male infertility by increasing the pregnancy rate and sperm kinetic features in humans. Therefore, carnitine diet supplementation could provide a potential therapy in improving the male reproductive ability [55].

\subsubsection{Protein}

Research findings on restricted protein intake in developing male rats have shown far reaching effects on spermatogenesis and epididymal sperm maturation (Table 6). Rats fed with 3\% protein revealed lower testosterone levels, inhibited spermatogenesis, immature testes, and delayed puberty compared to rats fed with $10 \%$ and $20 \%$ protein. The capability of protein deficient rats producing mature sperm was not completely impaired, but the initiation of spermatogenesis was retarded by 2.5 to 3 weeks. It is suggested that protein deficiency has an impact not only on normal gonadal organ function and spermatogenesis, but also on tubular atrophy. In a study

Table 6. The roles of macronutrients in sperm functions.

\begin{tabular}{|c|c|}
\hline Macronutrients & Roles in Sperm Functions \\
\hline Lipids: DHA & $\begin{array}{l}\text { Major component in sperm tail [52]. } \\
\text { Important in sperm motility and fertility capacity } \\
\text { [52]. }\end{array}$ \\
\hline Amino Acid: Arg & $\begin{array}{l}\text { Precursor of putrescine, spermidine and spermine } \\
\text { synthesis [14]. } \\
\text { Essential for spermatogenesis and sperm motility } \\
\text { [14]. }\end{array}$ \\
\hline Amino Acid: LC & $\begin{array}{c}\text { Enhances sperm motility, maturation and sper- } \\
\text { matogenesis [54]. } \\
\text { Supply energy to spermatozoa [54]. } \\
\text { Protect cell membrane and DNA against } \\
\text { ROS-induced damage [54,55]. }\end{array}$ \\
\hline
\end{tabular}

about effects of maternal protein restriction on the male reproductive system, protein deficiency also results in delayed testicular development and low sperm counts [13]. Compared to the group which received restricted protein diets either during fetal development or lactation, the group fed with inadequate protein balance from pregnancy to the weaning period displayed lower sperm counts without fertility dysfunction. Apparently, maternal protein restriction during development changes descendant testicular function and gonadal hormone secretion. In conclusion, protein deprivation depresses gonadotropin production, either through elevated testerone levels or LH levels, and negatively affects testicular development [13].

\section{DISCUSSION}

\subsection{The Main Nutrient Issues for High Quality Spermatogenesis}

Although there are many studies about the importance of nutrients during spermatogenesis, there are some aspects which are rarely understood and need deeper investigations. These aspects include how the nutritional elements influence spermatozoan morphology, physiological maturation and fertilization capacity.

\subsubsection{Can Nutrition Affect Sperm Morphology?}

It is known that zinc and selenium have a large impact on the morphological integrity of sperm, especially the midpiece formation. An abnormal midpiece will cause defects in the connection of sperm head and sperm tail. Studies have also proven that Vitamin E has a remarkable effect to restore incomplete acrosomal membranes. Since midpiece and acrosome are integral parts of sperm for successful fertilization, while some nutrition deficiencies cause midpiece and acrosome abnormalities, relevant further and deeper studies need to be done. It is also worth to ponder whether other nutritional factor deficiencies will cause sperm morphological defects besides zinc and selenium.

\subsubsection{What Are the Major Nutrients for Sperm Maturation in the Epididymis?}

The capability of sperm to move and fertilize eggs is acquired during the epididymal transit as various fluid contents in caput epididymis, corpus epididymis and caudal epididymis alters the surface of the sperm plasma membrane and provide them with new properties. Therefore, sperm motility and fertilization capability vary at different areas. As sperms reaches the caudal epididymis, the concentration of zinc diminishes, while the LC and calcium concentration increases. LC and calcium ions are the exogenous sources of sperm motility. Most of the studies discuss about how the contents in the caudal epididymis affect sperm capacity but the function of the 
caput epididymis and corpus epididymis are rarely mentioned, perhaps it is because the caudal epididymis is the crucial region where sperm obtains those key characteristics. Overall understanding of how the nutritional contents in each part of epididymis influence sperm motility and fertilization capacity will build a better foundation on further investigations about male infertility.

\subsubsection{Nutritional Environment for Capacitation and Hypo-Activity of Sperm before Fertilization}

Zinc plays a role in the ejaculate by inhibiting capacitation, while Selenium together with Vitamin E promotes the acrosome reaction. However, information about the key factors affecting sperm capacitation and hyperactivation after ejaculation is still scarcely known. Future studies should put emphasis on particular nutritional deficiencies that cause sperm to lose energy and ability to fuse with the egg and which nutritional factors promote or restrain capacitation and hyperactivation. A better understanding of these questions will lay a solid foundation for further investigations, especially beneficial to increase rate of success of in vitro fertilization.

\subsection{Prospect}

In conclusion, spermatogenesis is an extremely intricate process which requires strict and rigid conditions to form mature and healthy spermatozoa. One of the conditions needed to be fulfilled is an abundance of nutritional supplies which are crucial during spermatogenesis. During the initiation of spermatogenesis, folate and Vit $\mathrm{B}_{12}$ are especially critical due to their significant role in RNA and DNA synthesis. Selenium and zinc as well are also important co-factors which incorporate enzymes in the process of polymerization and transcription. GPX4 is also an essential element which works with selenium to protect vulnerable germ cells from oxidative stress. Vitamin A plays a role in regulating spermatogenesis, particularly during the starting phase. Both vitamin $\mathrm{B}_{9}$ and vitamin E deficiency negatively impacts sperm production. Vitamin C and Vitamin E both protect sperm from oxidative attack. These nutritional elements also play crucial roles in promoting sperm motility, semen quality, Sertoli cell and Leydig cell development. In addition, parts of the nutritional elements are involved in the process of capacitation and fertilization.

Molecular mechanisms of many nutritional elements involved in spermatogenesis are yet remained unclear. While the deleterious impacts of excessive amount of trace minerals and vitamins supplementation are not completely and clearly investigated. Researchers should focus more in future on experimenting and finding out the critical mechanisms of these elements in enhancing spermatogenesis and the definite concentration of these dietary intakes which are needed to reach optimum tes- ticular growth and spermatogenesis. A better understanding on the mechanisms of how these nutritional elements works on spermatogenesis and testicular functions may be conducive to treating male infertility, thereby contribute greatly to humankind. In addition, further investigations on adequate quantities of these trace minerals and vitamins to achieve maximal sexual development in mammals will lay a strong foundation for further studies on exploring the best combination of these nutritional elements with appropriate dosage in nutritional therapy to cure male infertility.

\section{ACKNOWLEDGEMENTS}

This work was supported in part by the following grants: National Natural Science Foundation of China, Grant number: No. 31072198 and 40776079; National Basic Research Program of China (973 Program), Grant number: No. 2007CB948104. The authors are also indebted to the members of the Sperm Laboratory for many helpful discussions.

\section{REFERENCES}

[1] Emanuele, M.A. and Emanuele, N.V. (1998) Alcohol's effects on male reproduction. Alcohol Health \& Research World, 22, 195-201.

[2] Foley, G.L. (2001) Overview of male reproductive pathology. Toxicologic Pathology, 29, 49-63. doi:10.1080/019262301301418856

[3] Hidiroglou, M. and Knipfel, J.E. (1984) Zinc in mammalian sperm: A review. Journal of Dairy Science, 67, 1147-1156. doi:10.3168/jds.S0022-0302(84)81416-2

[4] Chung, S.S.W., Wang, X. and Wolgemuth, D.J. (2009) Expression of retinoic acid receptor alpha in the germline is essential for proper cellular association and spermiogenesis during spermatogenesis. Development, 136, 20912100. doi: $10.1242 /$ dev.020040

[5] Abdu, S.B. (2008) Effect of vitamins deficiencies on the histological structure of the testis of albino mice Mus musculus. Saudi Journal of Biological Sciences, 15, 269278.

[6] Chinoy, N.J. and Ahmedabad, A.S. (1998) Amelioration of fluoride toxicity by vitamins $\mathrm{E}$ and $\mathrm{D}$ in reproductive functions of male mice. Fluoride, 31, 203-216.

[7] Ebisch, I.M.W., Thomas, C.M.G., Peters, W.H.M., Braat, D.D.M. and Steegers-Theunissen, R.P.M. (2007) The importance of folate, zinc and antioxidants in the pathogenesis and prevention of subfertility. Human Reproduction Update, 13, 163-174. doi:10.1093/humupd/dml054

[8] Hawkes, W.C. and Turek, P.J. (2001) Effects of dietary selenium on sperm motility in healthy men. Journal of Andrology, 22, 764-772.

[9] Yokoi, K., Uthus, E.O. and Nielsen, F.H. (2003) Nickel deficiency diminishes sperm quantity and movement in rats, Biological Trace Element Research, 93, 141-153. doi:10.1385/BTER:93:1-3:141

[10] Lee, B., Pine, M., Johnson, L., Rettori, V., Hiney, J.K. and Dees, W.L. (2006) Manganese acts centrally to activate reproductive hormone secretion and pubertal devel- 
opment in male rats. Reproductive Toxicology, 22, 580585. doi:10.1016/j.reprotox.2006.03.011

[11] Tuormaa, T.E. (2000) Chromium, selenium and copper and other trace minerals in health and reproduction. Journal of Orthomolecular Medicine, 15, 145-157.

[12] Aksoy, Y., Aksoy, H., Altinkaynak, K., Aydin, H.R. and Ozkan, A. (2006) Sperm fatty acid composition in subfertile men. Prostaglandins, Leukotrienes and Essential Fatty Acids, 75, 75-79. doi:10.1016/j.plefa.2006.06.002

[13] Zambrano, E., Rodríguez-González, G.L., Guzman, C., García-Becerra, R., Boeck, L., Díaz, L., Menjivar, M., Larrea, F. and Nathanielsz, P.W. (2005) A maternal low protein diet during pregnancy and lactation in the rat impairs male reproductive development. Journal of Physiology, 563, 275-284.

[14] Wu, G., Bazer, F.W., Davis, T.A., Kim, S.W., Li, P., Marc Rhoads, J., Satterfield, M.C., Smith, S.B., Spencer, T.E. and Yin, Y. (2009) Arginine metabolism and nutrition in growth, health and disease. Amino Acids, 37, 153-168. doi:10.1196/annals.1320.017

[15] Ng, C.M., Blackman, M.R., Wang, C. and Swerdloff, R.S. (2004) The role of carnitine in the male reproductive system. Annals New York Academy of Sciences, 1033, 177-188. doi:10.1196/annals.1320.017

[16] Jones, R.C. (1999) To store or mature spermatozoa? The primary role of the Epididymis. International Journal of Andrology, 22, 57-67. doi:10.1046/j.1365-2605.1999.00151.x

[17] Mason, K.E., Burns, W.A. and Smith Jr., J.C. (1982) Testicular damage associated with zinc deficiency in preand postpubertal rats: Response to zinc repletion. The Journal of Nutrition, 112, 1019-1028.

[18] Wong, W.Y., Flik, G., Groenena, P.M., Swinkels, D.W., Thomas, C.M., Copius-Peereboom, J.H.J., Merkus, H.M.W.M. and Steegers-Theunissen, R.P.M. (2001) The impact of calcium, magnesium, zinc, and copper in blood and seminal plasma on semen parameters in men. Reproductive Toxicology, 15, 131-136. doi:10.1016/S0890-6238(01)00113-7

[19] Oliveira, C.E.A., Badú, C.A., Ferreira, W.M., Kamwa, E.B. and A.M.Q. Lana, (2004) Effects of dietary zinc supplementation on spermatic characteristics of rabbit breeders. 8th World Rabbit Congress, Mexico, 7-10 September 2004, 315-321.

[20] Liu, D.Y., Sie, B.S., Liu, M.L., Agresta, F. and Baker, H.W.G. (2009) Relationship between seminal plasma zinc concentration and spermatozoa-zona pellucida binding and the ZP-induced acrosome reaction in subfertile men. Asian Journal of Andrology, 11, 499-507. doi:10.1038/aja.2009.23

[21] Batra, N., Nehru, B. and Bansal, M.P. (2004) Reproductive potential of male Portan rats exposed to various levels of lead with regard to zinc status. British Journal of Nutrition, 91, 387-391. doi:10.1079/BJN20031066

[22] Kaur, P. and Bansal, M.P. (2005) Effect of seleniuminduced oxidative stress on the cell kinetics in testis and reproductive ability of male mice. Nutrition, 21, 351-357. doi:10.1016/j.nut.2004.05.028

[23] Beckett, G.J. and Arthur, J.R. (2005) Selenium and endocrine systems. Journal of Endocrinology, 84, 455-465. doi:10.1677/joe.1.05971

[24] Hadaszadeh, B.M. and Beggs, A.H. (2006) Selenopro- teins and their impact on human health through diverse physiological pathways. Physiology, 21, 307-315. doi:10.1152/physiol.00021.2006

[25] Schriever, S.C., Barnes, K.M., Evenson, J.K., Raines, A.M. and Sunde, R.A. (2009) Selenium requirements are higher for glutathione peroxidase-1 mRNA than gpx1 activity in rat testis. Experimental Biology and Medicine (Maywood), 234, 513-521. doi:10.3181/0812-RM-369

[26] Ebisch, I.M., Peters, W.H., Thomas, C.M., Wetzels, A.M., Peer, P.G. and Steegers-Theunissen, R.P. (2006) Homocysteine, glutathione and related thiols affect fertility parameters in the (sub)fertile couple. Human Reproduction, 21, 1725-1733. doi.org/10.1093/humrep/del081

[27] Young, S.S., Eskenazi, B., Marchetti, F.M., Block, G. and Wyrobek, A.J., (2008) The association of folate, zinc and antioxidant intake with sperm aneuploidy in healthy non-smoking men. Human Reproduction, 23, 1014-1022. doi:10.1093/humrep/den036

[28] Wang, S., Wang, G., Barton, B.E., Murphy, T.F. and Huang, H.F. (2007) Beneficial effects of vitamin E in sperm functions in the rat after spinal cord injury. Journal of Andrology, 28, 334-341. doi:10.2164/jandrol.106.001164

[29] Begum, H., Moniruddin, A.B.M. and Nahar, K. (2009) Environmental and nutritional aspect in male infertility. Journal of Medicine, 10, 16-19.

[30] Kann, M.L. and Serres, C. (1980) Development and Initiation of sperm motility in the hamster epididymis. $R e$ production Nutrition Development, 20, 1739-1749. doi:10.1051/rnd:19801001

[31] Schneider, M., Förster, H., Boersma, A., Seiler, A., Wehnes, H., Sinowatz, F., Neumüller, C., Deutsch, M.J., Walch, A., Hrabé de Angelis, M., Wurst, W., Ursini, F., Roveri, A., Maleszewski, M., Maiorino, M. and Conrad, M. (2009) Mitochondrial glutathione peroxidase 4 disruption causes male infertility. Faseb Journal, 23, 32333242. doi:10.1096/fj.09-132795

[32] Olson, G.E., Winfrey, V.P., Hill, K.E. and Burk, R.F. (2004) Sequential development of flagellar defects in spermatids and epididymal spermatozoa of selenium-deficient rats, Reproduction, 127, 335-342. doi:10.1530/rep.1.00103

[33] Tareq, K.M.A., Miah, A.G., Salma, U., Yoshida, M. and Tsujii, H. (2010) Effect of selenium and vitamin E on acrosome reaction in porcine spermatozoa. Reproduction of Medicine Biology, 9, 73-81. doi:10.1007/s12522-009-0041-x

[34] Suleiman, S.A., Ali, M.E., Zaki, Z.M., El-Malik, E.M. and M.A. Nasr, (1996) Lipid peroxidation and human sperm motility: Protective role of vitamin E. Journal of Andrology, 17, 530-537.

[35] Visconti, P.E., Ning, X., Fornés, M.W., Alvarez, J.G., Stein, P., Connors, S.A. and Kopf, G.S. (1999) Cholesterol efflux-mediated signal transduction in mammalian sperm: Cholesterol release signals an increase in protein tyrosine phosphorylation during mouse sperm capacitation. Developmental Biology, 214, 429-443. doi:10.1006/dbio.1999.9428

[36] Kothari, S., Thompson, A., Agarwalm, A. and du Plessis, S.S. (2010) Free radicals: Their beneficial and detrimental effects on sperm function. Indian Journal of Experimental Biology, 48, 425-435. 
[37] Uhland, A.M., Kwiecinski, G.G. and DeLuca, H.F. (1992) Normalization of serum calcium restores fertility in vitamin D-deficient male rats. Journal of Nutrition, 122, 1338-1344.

[38] Sönmez, M., Türk, G. and Yüce, A. (2005) The effect of ascorbic acid supplementation on sperm quality, lipid peroxidation and testosterone levels of male Wistar rats. Theriogenology, 63, 2063-2072. doi:10.1016/j.theriogenology.2004.10.003

[39] Yue, D., Yan, L., Luo, H., Xu, X. and Jin, X. (2010) Effect of Vitamin E supplementation on semen quality and the testicular cell membranal and mitochondrial antioxidant abilities in Aohan fine-wool sheep. Animal Reproduction Science, 118, 217-222. doi:10.1016/j.anireprosci.2009.08.004

[40] Internet Available: http://en.wikipedia.org/wiki/Semen

[41] Bleau, G., Lemarbre, J., Faucher, G., Roberts, K.D. and Chapdelaine, A. (1984) Semen selenium and human fertility. Fertility \& Sterility, 42, 890-894.

[42] Audet, I., Bérubé, N., Bailey, J.L., Laforest, J.P. and Matte, J.J. (2009) Effects of dietary vitamin supplementation and semen collection frequency on reproductive performance and semen quality in boars. Journal of Animal Science, 87, 1960-1970. doi:10.2527/jas.2008-1714

[43] Miller, E.R. Pastor-Barriuso, R., Dalal, D., Riemersma, R.A., Appel, L.J. and Guallar, E. (2005) Meta-analysis: High-dosage vitamin E supplementation may increase all-cause mortality. Annals of Internal Medicine, 142, 37-46. .

[44] Gliozzi, T.M., Zaniboni, L., Maldjian, A., Luzi, F., Maertens, L. and Cerolini, S. (2009) Quality and lipid composition of spermatozoa in rabbits fed DHA and vitamin E rich diets. Theriogenology, 71, 910-919. doi:10.1016/j.theriogenology.2008.10.022

[45] Bolle, P., Evandri, M.G. and Saso, L. (2002) The controversial efficacy of vitamin $\mathrm{E}$ for human male infertility. Contraception, 6, 313-315. doi:10.1016/S0010-7824(02)00277-9

[46] Brito, L.F., Barth, A.D., Rawlings, N.C., Wilde, R.E., Crews Jr, D.H., Mir, P.S. and Kastelic, J.P. (2007) Effect of improved nutrition during calfhood on serum metabolic hormones, gonadotropins, and testosterone concentrations, and on testicular development in bulls. Domestic Animal Endocrinology, 33, 460-469. doi:10.1016/j.domaniend.2006.09.004

[47] Davies, D.V., Mann, T. and Rowson, L.E. (1957) Effect of nutrition on the onset of male sex hormone activity and sperm formation in monozygous bull-calves. Proceedings of the Royal Society of London Series B-Biological Sciences, 147, 332-351. doi:10.1098/rspb.1957.0054

[48] Almeida, A.M., Schwalbach, L.M.J., Cardoso, L.A. and Greyling, J.P.C. (2007) Scrotal, testicular and semen characteristics of young Boer bucks fed winter veld hay: The effect of nutritional supplementation. Small Ruminant Research, 73, 216-220. doi:10.1016/j.smallrumres.2007.02.001

[49] Conquer, J.A., Martin, J.B., Tummon, I., Watson, L. and Tekpetey, F. (2000) Effect of DHA supplementation on DHA status and sperm motility in asthenozoospermic males. Lipids, 35, 149-154. doi:10.1007/BF02664764

[50] Zalata, A.A., Christophe, A.B., Depuydt, C.E., Schoonjans, F. and Comhaire, F.H. (1998) The fatty acid composition of phospholipids of spermatozoa from infertile patients. Molecular Human Reproduction, 4, 111-118. doi:10.1093/molehr/4.2.111

[51] Rooke, J.A., Shao, C.C. and Speake, B.K. (2001) Effects of feeding tuna oil on the lipid composition of pig spermatozoa and in vitro characteristics of semen. Reproduction, 121, 315-322. .doi:10.1530/rep.0.1210315

[52] Lin, D.S., Neuringer, M. and Connor, W.E. (2004) Selective changes of docosahexaenoic acid-containing phospholipid molecular species in monkey testis during puberty. Journal of Lipid Research, 45, 529-535. doi:10.1194/jlr.M300374-JLR200

[53] Brinsko, S.P., Varner, D.D., Love, C.C., Blanchard, T.L., Day, B.C. and Wilson, M.E. (2005) Effect of feeding a DHA-enriched nutriceutical on the quality of fresh, cooled and frozen stallion semen. Theriogenology, 63, 1519-1527. doi:10.1016/j.theriogenology.2004.07.010

[54] Jeulin, C. and Lewin, L.M. (1996) Role of free L-carnitine and acetyl-L-carnitine in post-gonadal maturation of mammalian spermatozoa. Human Reproduction Update, 2, 87-102. doi:10.1093/humupd/2.2.87

[55] Zhou, X., Liu. F. and Zhai, S. (2007) Effect of L-carnitine and/or L-acetyl-carnitine in nutrition treatment for male infertility: A systematic review. Asia Pacific of Journal Clinical Nutrition, 16, 383-390. 